\title{
A NOTE ON DISCRETE-TIME DYNAMICAL SYSTEMS UNDER PERIODIC PERTURBATION
}

HUIMIN LI AND XIAOSONG YANG

Received 11 December 2005; Accepted 27 February 2006

We present some results on the existence and the minimum period of periodic orbits for discrete-time dynamical systems under periodic perturbation. Some examples are presented to illustrate these results.

Copyright (c) $2006 \mathrm{H}$. Li and X. Yang. This is an open access article distributed under the Creative Commons Attribution License, which permits unrestricted use, distribution, and reproduction in any medium, provided the original work is properly cited.

\section{Introduction}

Consider the following discrete-time dynamical systems:

$$
x(n+1)=f(x(n), y(n)), \quad x \in \mathbb{R}^{n}, y \in \mathbb{R}^{m},
$$

where $f$ is a continuous function, $y(n)$ is a perturbation function.

We are interested in the following question.

Question 1.1. Under what conditions system (1.1) has a periodic orbit when the perturbation is a periodic signal?

There have been a lot of researches in the literature on the periodic trajectories of discrete-time dynamical systems [1, 3-15]. However, to the authors' best knowledge, the existence of periodic orbits in a discrete-time dynamical system with a periodic perturbation has not received enough investigations, therefore in this short paper we study the above question.

\section{Main results}

In this section, we present some results on the existence and the minimum period of periodic orbits of system (1.1) under the following assumptions. 
Assumption 2.1. The perturbation $y(n)$ is assumed to be a periodic signal of minimum period $k$ satisfying $y_{i}=y_{i+k}$, that is, the periodic perturbation is $\left\{y_{0}, y_{1}, \ldots, y_{k-1}\right\}$, where $i$ is a nonnegative integer and $k$ is a positive integer.

Assumption 2.2. $f\left(0, y_{i}\right) \neq 0$ provided that $y_{i} \neq 0, i=0,1, \ldots, k-1$.

Assumption 2.3. We have

$$
\varlimsup_{\|x\| \rightarrow \infty} \frac{\left\|f\left(x, y_{i}\right)\right\|}{\|x\|}<1, \quad i=0,1, \ldots, k-1
$$

Before stating our results, we first recall a fixed-point theorem due to Brower, which is useful in the following arguments.

Lemma 2.4 (Brower fixed-point theorem). Let $F: C \subset \mathbb{R}^{n} \rightarrow C \subset \mathbb{R}^{n}$ be a continuous mapping, where $C$ is a nonempty, bounded, close and convex set. Then $F$ has a fixed point. Its proof can be found in [2].

Now we present the following theorems.

Theorem 2.5. Suppose that Assumptions 2.1, 2.2, and 2.3 hold. Then system (1.1) has a nontrivial periodic orbit.

Proof. According to Assumption 2.1 we know that the periodic signal is $\left\{y_{0}, y_{1}, \ldots\right.$, $\left.y_{k-1}\right\}$ with minimum period $k$ satisfying $y_{i}=y_{i+k}$, where $i$ is a nonnegative integer and $k$ is a positive integer.

Since $\varlimsup_{\|x\| \rightarrow \infty}\left(\left\|f\left(x, y_{i}\right)\right\| /\|x\|\right)<1$, for every $y_{i}, i=0,1, \ldots, k-1$, there exist corresponding positive numbers $m_{i}, i=0,1, \ldots, k-1$ such that $\left\|f\left(x, y_{i}\right)\right\|<\|x\|$ when $\|x\|>$ $m_{i}$.

Let $\overline{m_{i}}=\max _{\|x\| \leq m_{i}}\left\|f\left(x, y_{i}\right)\right\|>0$, let $M_{i}=\max \left(m_{i}, \overline{m_{i}}\right)$, and let $M=\max \left\{M_{i}, i=\right.$ $0,1, \ldots, k-1\}>0$. It is easy to see that if $\|x\| \leq M$, then for every $y_{i}, i=0,1, \ldots, k-1$, $\left\|f\left(x, y_{i}\right)\right\| \leq M$, which implies that the set $U=\{x \mid\|x\| \leq M\}$ is a positive invariant set of the following systems:

$$
x(n+1)=f\left(x(n), y_{i}\right), \quad i=0,1, \ldots, k-1 .
$$

Let $f_{i}=f\left(x, y_{i}\right), i=0,1, \ldots, k-1$, and let $F=f_{k-1} \circ \cdots \circ f_{0}$. Then $F: U \rightarrow U$ is a continuous mapping. By Brower fixed-point theorem, the mapping $F$ has a fixed point $\bar{x}_{0}$, that is, $F\left(\bar{x}_{0}\right)=\bar{x}_{0}, f_{k-1} \circ \cdots \circ f_{0}\left(\bar{x}_{0}\right)=\bar{x}_{0}$.

Let $\bar{x}_{i}=f_{i-1}\left(\bar{x}_{i-1}\right)$, we get $f_{k-1}\left(\bar{x}_{k-1}\right)=\bar{x}_{0}$, thus the orbit $o\left(\bar{x}_{0}\right)=\left\{\bar{x}_{0}, \bar{x}_{1}, \ldots, \bar{x}_{k-1}\right\}$ is a periodic orbit. This implies that $o\left(\bar{x}_{0}\right)$ contains a periodic orbit whose minimum period $j$ divides $k$, where $j$ is a positive integer.

In addition, according to Assumption 2.2, we have that there exists $i_{0} \in\{0,1, \ldots, k-1\}$ such that $\bar{x}_{i_{0}} \neq 0$. This shows that $o\left(\bar{x}_{0}\right)$ contains a nontrivial periodic orbit. The proof is complete. 
It seems that the period of $o\left(\bar{x}_{0}\right)$ should not be less than that of the perturbation $y(n)$, which is often the case when dynamical systems described by ordinary differential equations are forced by periodic signals. However, the following example shows that this can not be the case in discrete-time systems.

Consider the following example.

Example 2.6. We have

$$
x(n+1)=f(x(n), y(n)), \quad x \in \mathbb{R}, y \in \mathbb{R},
$$

where $f(x, y)=x /(2+|y|)+|y| /(2+|y|)$, the perturbation $y(n)$ is a periodic signal $\{1,-$ $1 / 2,-1,1 / 2\}$.

It is easy to see that for Example 2.6 the conditions stated in Theorem 2.5 are satisfied. Thus according to Theorem 2.5, system (2.3) has a periodic orbit. In fact, it can be verified that the orbit $\{5 / 13,6 / 13\}$ is a periodic orbit of $(2.3)$.

Remark 2.7. In Example 2.6, we have the following facts.

(a) The system $x(n+1)=f(x(n), 0)=(1 / 2) x(n)$ is globally asymptotically stable which implies that the system does not have nontrivial periodic orbits. However, its perturbed system has a nontrivial periodic orbit.

(b) The functions $f_{i}=f\left(x, y_{i}\right), i=0,1,2,3$, satisfy the following condition:

$$
f_{3} \circ f_{2} \circ f_{1} \circ f_{0}(x)=f_{1} \circ f_{0}(x)
$$

which shows that $f_{3} \circ f_{2} \circ f_{1} \circ f_{0}(x)$ and $f_{1} \circ f_{0}(x)$ have the same fixed points.

(c) The minimum period of the periodic orbit of the perturbation is 4 , but the minimum period of its corresponding periodic orbit is 2 , which defies our intuition.

Considering the above fact (c), we want to know under what conditions (1.1) has a nontrivial $k$-period orbit. This will be given in Theorem 2.8. Before presenting Theorem 2.8, we assume that the integer $k$ (in Assumption 2.1) has the following factors: $k_{1}, \ldots, k_{r}$ and $0<k_{1}<k_{2}<\cdots<k_{r}<k$, where $r$ and $k_{1}, \ldots, k_{r}$ are positive integers and $k_{l}$ divides $k$, $l=1,2, \ldots, r$. Moreover, we let $f_{i}=f\left(x, y_{i}\right), F_{k_{l}}=f_{k_{l}-1} \circ \cdots \circ f_{0}, F_{2 k_{l}}=f_{2 k_{l}-1} \circ \cdots \circ f_{0}$.

Theorem 2.8. Suppose that Assumptions 2.1, 2.2, and 2.3 hold, and for any $k_{l} \in\left\{k_{1}, \ldots, k_{r}\right\}$, $F_{k_{l}}$ and $F_{2 k_{l}}$ do not have the same fixed points. Then system (1.1) also has a periodic orbit of minimum period $k$.

Proof. In terms of the proof of Theorem 2.5, we have that system (1.1) has a periodic orbit $\left\{\bar{x}_{0}, \bar{x}_{1}, \ldots, \bar{x}_{j-1}\right\}$ of minimum period $j$, where $j \in\left\{k_{1}, \ldots, k_{r}, k\right\}$. Below, our purpose is to prove that the minimum period of the above periodic orbit is $k$, that is, $j=k$. For this purpose, we assume that $j \in\left\{k_{1}, \ldots, k_{r}\right\}$. Since $\left\{\bar{x}_{0}, \bar{x}_{1}, \ldots, \bar{x}_{j-1}\right\}$ is a periodic orbit, it is evident that $\bar{x}_{0}$ is the fixed point of $F_{j}$ and $F_{2 j}$, which is a contradiction to the assumption of Theorem 2.8. Therefore the minimum period of the periodic orbit $\left\{\bar{x}_{0}, \bar{x}_{1}, \ldots, \bar{x}_{j-1}\right\}$ is also $k$. The proof is complete. 
4 Discrete-time systems under periodic perturbation

We apply Theorem 2.8 to discuss the following system.

Example 2.9. We have

$$
x(n+1)=f(x(n), y(n)), \quad x \in \mathbb{R}, y \in \mathbb{R},
$$

where $f(x, y)=x /(2+|y|)+y /(2+|y|)$, the perturbation $y(n)$ is a periodic signal $\{1,-$ $1 / 2,-1,1 / 2\}$.

It is easy to verify that in Example 2.9 the conditions stated in Theorem 2.8 are satisfied. Thus by Theorem 2.8, we know that system (2.5) only has a periodic orbit of period 4. It is easy to verify that the period orbit is $\{1 / 17,6 / 17,-1 / 17,-6 / 17\}$.

\section{Acknowledgment}

The research is partially supported by Talents Foundation of Huazhong University of Science and Technology (0101011092), and is partially supported by Program for New Century Excellent Talents in the university.

\section{References}

[1] R. M. Abu-Saris and Q. M. Al-Hassan, On global periodicity of difference equations, Journal of Mathematical Analysis and Applications 283 (2003), no. 2, 468-477.

[2] R. P. Agarwal, M. Meehan, and D. O'Regan, Fixed Point Theory and Applications, Cambridge Tracts in Mathematics, vol. 141, Cambridge University Press, Cambridge, 2001.

[3] F. M. Atici and G. Sh. Guseinov, Positive periodic solutions for nonlinear difference equations with periodic coefficients, Journal of Mathematical Analysis and Applications 232 (1999), no. 1, 166182.

[4] C. T. H. Baker and Y. Song, Periodic solutions of discrete Volterra equations, Mathematics and Computers in Simulation 64 (2004), no. 5, 521-542.

[5] Y. Chen, All solutions of a class of difference equations are truncated periodic, Applied Mathematics Letters 15 (2002), no. 8, 975-979.

[6] S. Elaydi and R. J. Sacker, Global stability of periodic orbits of non-autonomous difference equations and population biology, Journal of Differential Equations 208 (2005), no. 1, 258-273.

[7] J. Feuer, Periodic solutions of the Lyness max equation, Journal of Mathematical Analysis and Applications 288 (2003), no. 1, 147-160.

[8] J. A. C. Gallas, On the origin of periodicity in dynamical systems, Physica A. Statistical Mechanics and Its Applications 283 (2000), no. 1-2, 17-23.

[9] M. K. Kinyou and A. A. Sagle, Quadratic dynamical systems and algebras, Journal of Differential Equations 117 (1995), no. 1, 67-126.

[10] P. Misra, Time-invariant representation of discrete periodic systems, Automatica 32 (1996), no. 2, 267-272.

[11] H. Sedaghat, Periodicity and convergence for $x_{n+1}=\left|x_{n}-x_{n-1}\right|$, Journal of Mathematical Analysis and Applications 291 (2004), no. 1, 31-39.

[12] S. Stevo, The recursive sequence $x_{n+1}=g\left(x_{n}, x_{n-1}\right) /\left(A+x_{n}\right)$, Applied Mathematics Letters 15 (2002), no. 3, 305-308.

[13] H. D. Voulov, On the periodic nature of the solutions of the reciprocal difference equation with maximum, Journal of Mathematical Analysis and Applications 296 (2004), no. 1, 32-43. 
[14] Y. Wang and J. Jiang, The general properties of discrete-time competitive dynamical systems, Journal of Differential Equations 176 (2001), no. 2, 470-493.

[15] Z. Zhou, Periodic orbits on discrete dynamical systems, Computers \& Mathematics with Applications 45 (2003), no. 6-9, 1155-1161.

Huimin Li: Department of Mathematics, Huazhong University of Science and Technology, Wuhan 430074, China

Xiaosong Yang: Department of Mathematics, Huazhong University of Science and Technology, Wuhan 430074, China

E-mail address: yangxs@cqupt.edu.cn 


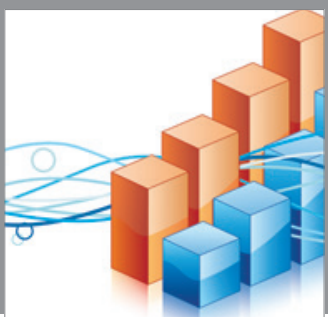

Advances in

Operations Research

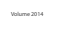

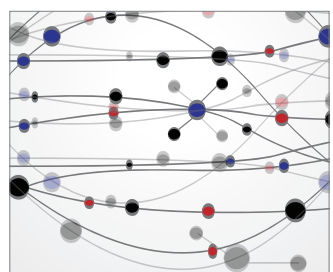

\section{The Scientific} World Journal
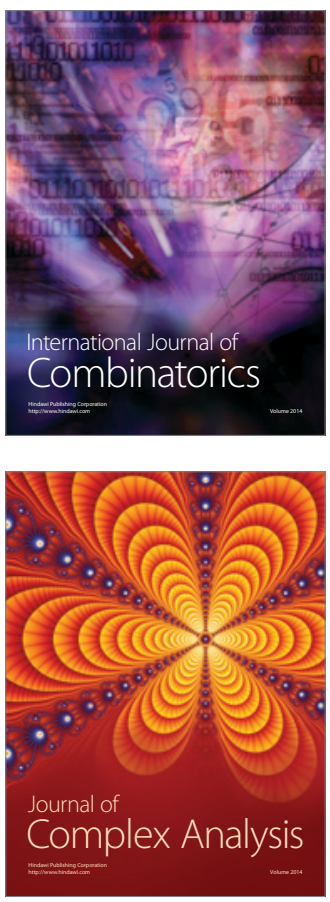

International Journal of

Mathematics and

Mathematical

Sciences
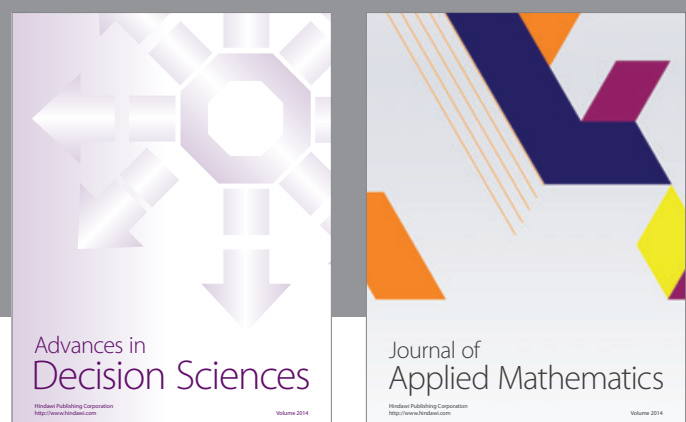

Journal of

Applied Mathematics
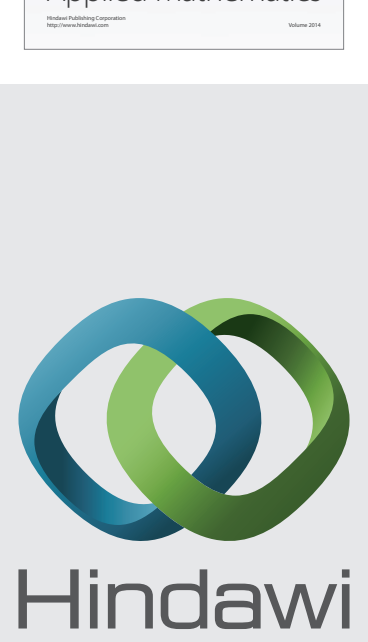

Submit your manuscripts at http://www.hindawi.com
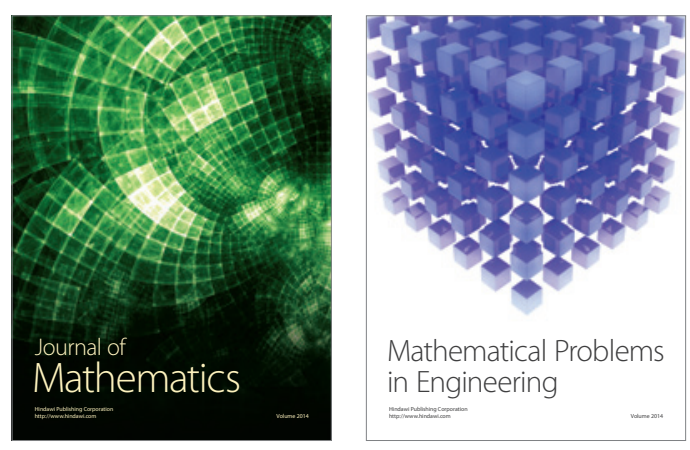

Mathematical Problems in Engineering
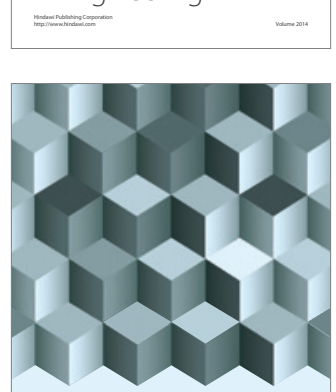

Journal of

Function Spaces
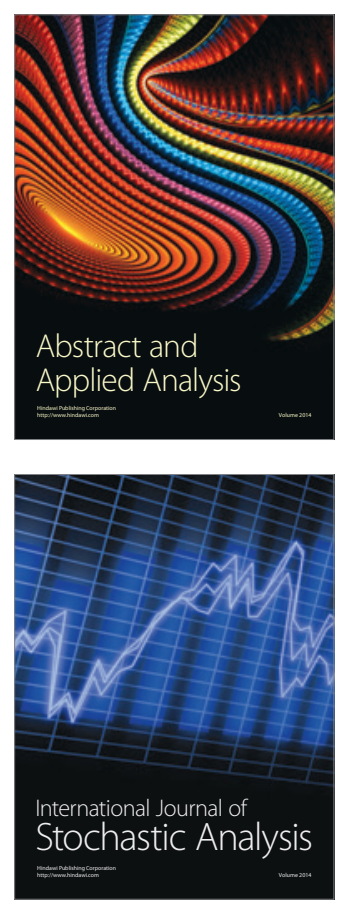

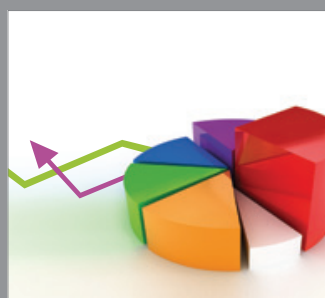

ournal of

Probability and Statistics

Promensencen
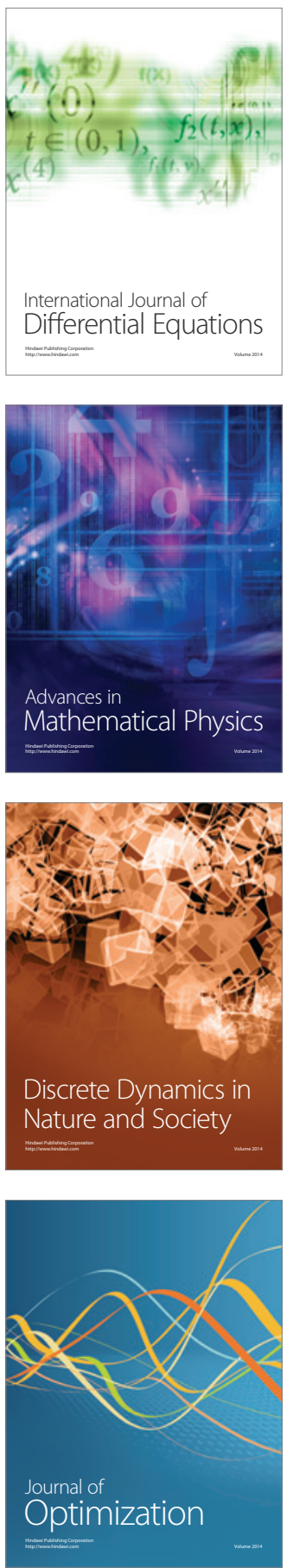